\title{
Medizinische Probleme mit Neophyten?
}

\author{
Neophyten sind Adventivpflanzen, die etwa seit dem 16. Jahrhundert - nach der Entdeckung der Neuen Welt - \\ fester Bestandteil unserer Flora geworden sind
}

\author{
R. Widmer-Bösch
}

Korrespondenz:

Rudolf Widmer-Bösch Wissegg 1

CH-9043 Trogen
Neophyten sind «Neubürger» in unserer Vegetation. Es sind nicht spontane Neuschöpfungen! Sie sind von Menschen absichtlich oder unabsichtlich in unsere Flora verschleppt worden, also «Hergekommene» oder eben Adventivpflanzen. Meist treten sie nur vorübergehend auf Bahnarealen, Märkten, Aufschüttungen, Äckern usw. auf und können sich nicht dauerhaft etablieren. Adventiv sind auch Pflanzen, die erst in neuerer Zeit zu uns gelangt sind, zum Beispiel als Früchte von Vögeln oder als Samen mit dem Wind hergebracht. Solche stammen oft aus dem Süden. Viele davon leben nur kurze Zeit in unserem Klima und wenige wurden integriert, wie etwa das Zimbelkraut.

Im folgenden sind einige Pflanzen der Schweiz genannt, die aus irgendeinem fernen Kontinent $\mathrm{zu}$ uns gelangt sind und sich selbständig - ohne unser Zutun - vermehren. Gartenblumen und Parkbäume wie etwa die Tulpen oder der Mammutbaum fallen nicht unter die Bezeichnung «Neophyten», weil sie sich nicht selbständig in die Wildflora ausbreiten. Pflanzen, die schon zur Zeit der Römer zu uns gelangt sind - etwa der Buchsbaum oder die Edelkastanie im Tessin -, gehören auch nicht zu den Neophyten im engeren Sinn.

Die gestellte Frage ist, ob sich innerhalb unserer Neophytenflora Arten finden, die Mediziner beschäftigen könnten, sei es wegen Vergiftungen oder zu phytotherapeutischen Zwecken.

Einige Beispiele von Neophyten:

\section{Wasserpest (Elodea canadensis)}

Dieses ursprünglich nordamerikanische «Wasserunkraut» (aquatic weed) soll aus botanischen Gärten in Berlin entflohen sein. Ab 1869 wurde es in Deutschland zum Problem, weil es Gewässer verunkrautete. In der Schweiz findet man die Wasserpest heute nicht selten in stehenden und langsam fliessenden Gewässern. Im Biologieunterricht nutzt man Elodea gerne, um unter dem Mikroskop die rotierende Bewegung der Chlorophyllkörper zu beobachten. Medizinisch hat die Wasserpest keine Bedeutung.

\section{Japanischer Staudenknöterich und Springkräuter}

Der Japanische Staudenknöterich (Reynoutria japonica) aus dem Himalaja und die zwei neuen Springkräuter (Impatiens glandulifera und Impatiens parviflora), beide aus Asien, haben sich in den letzten Jahren enorm ausgebreitet. Beide sind pharmakologisch unbedeutsam.

\section{Kanadische Goldrute (Solidago canadensis)} In der Schweiz wachsen vier Arten von Goldruten. Die Echte Goldrute (Solidago virgaurea) ist in Europa wahrscheinlich ursprünglich. Die andern, Solidago gigantea, Solidago canadensis und Solidago graminifolia (Kt. Schaffhausen), stammen aus Nordamerika und überwuchern Auenwälder, Ufergebüsche, Waldlichtungen und Ödland. Die heimische Goldrute wurde schon im Mittelalter als mässiges Diuretikum verwendet und kommt noch heute in zahlreichen Fertigpräparaten vor. Die amerikanischen Arten sind weder Gift- noch Heilpflanzen.

\section{Riesenbärenklau \\ (Heracleum montegazzianum)}

Erst seit etwa 1980 breitet sich dieser bis über drei Meter hohe Bärenklau - er heisst botanisch Montegazzis Bärenklau - in der Schweiz aus. Er stammt aus dem Kaukasus. Bei Berührung verursacht die Pflanze eine Fotosensibilisierung der Haut, die durch Sonnenbestrahlung zu Verbrennungen führen kann. $\mathrm{Zu}$ besonders schweren Hautentzündungen kommt es, wenn beim Abschlagen der Stengel austretender Saft auf die Haut tropft.

\section{Traubenkraut, Ambrosie \\ (Ambrosia artemisiifolia)}

Dieser aus Amerika stammende Korbblüher tritt in letzter Zeit immer häufiger auf, meist auf Äckern, an Wegrändern und auf Schuttplätzen. Seine Samen sind gelegentlich in Vogelfutter enthalten und werden durch den Transport von Erdreich mit Fahrzeugen verbreitet. Ob die Klimaerwärmung, wie dies oft behauptet wird, auch schuld an der Ausbreitung ist, sei dahingestellt. 


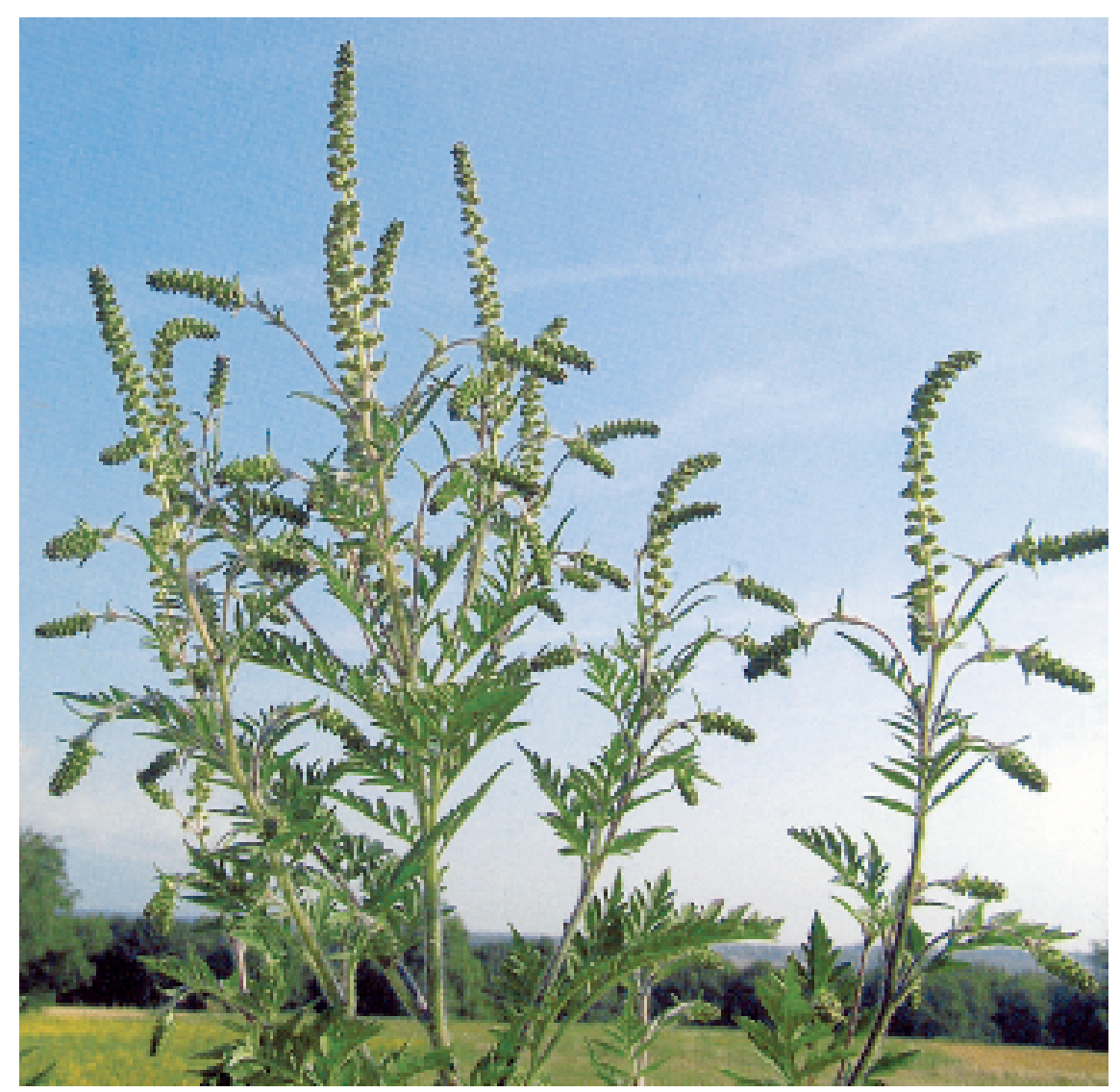

Ambrosia: eine gefährliche Pflanze. bekannt. Über die positiven und negativen Wirkungen unreifer Mohnköpfe (Opium) muss in einer Ärztezeitung kaum gerätselt werden.

\section{Amerikanischer Lebensbaum (Thuja occidentalis)}

Der aus Nordamerika stammende Baum wird oft als Heckenstrauch oder Parkbaum kultiviert. Er kann aber hin und wieder verwildern. Es wird behauptet, der Name «Lebensbaum» stamme daher, weil sich Seefahrer mit den schuppenförmigen Blättern vor Skorbut schützten. Auf die Menge kommt es an! Der Baum enthält ein ätherisches Öl mit stark reizender Wirkung. Nach peroraler Aufnahme führt es zu schwersten Intoxikationen mit langanhaltenden Krämpfen und degenerativen Veränderungen der Leber, Nierenschäden und Blutungen der Magenschleimhaut. Ursache der Giftigkeit ist vor allem das Thujon, dessen Gefährlichkeit auch vom ätherischen Öl im Absinth bekannt ist.

Der Neophyten sind noch viele. Als Heil- oder Giftpflanzen spielen sie meist keine Rolle. Einzig vor dem Riesenbärenklau und der Ambrosia muss man sich hüten. Bedeutsamer für die Medizin sind fremde Gartenpflanzen: Beeren der Kartoffel oder deren grüne Knollen, rohe Bohnen, Goldregen, Oleander usw. Als Volksheilmittel wird zum Beispiel der aus Amerika stammende Rote Sonnenhut (Echinacea) angepriesen, wobei seine Wirkung umstritten ist.

Die Zahl der phytotherapeutisch genutzten oder als Giftpflanzen gefürchteten Arten ist weitaus häufiger in der einheimischen Vegetation zu finden. Medikamente wie solche aus Sägepalmenfrüchten, Ginkgoblättern, Ginsengwurzeln usw. stammen nicht aus europäischem Anbau. In der Tiermedizin ist es nicht anders: Das gefürchtete und heute zu bekämpfende Jakobskreuzkraut (Senecio jacobaea) ist kein Neophyt. Es wächst seit Menschengedenken bei uns. Von den eingeführten Grasarten gehen weder Gefahren noch bekannte Heilwirkungen aus.

\section{Schlafmohn (Papaver somnifera)}

Mehr lila als rot blüht der Schlafmohn, der da und dort angebaut wird und gelegentlich verwildert. Seine ursprüngliche Heimat ist nicht 